\title{
Interaction between Relativistic Electrons and Mesoscopic Plasmonic Tapers
}

Surong Guo ${ }^{1}$, Nahid Talebi ${ }^{1}$, Wilfried Sigle ${ }^{1}$, Christoph Lienau ${ }^{2}$, Alfredo Campos ${ }^{3}$, Mathieu Kociak ${ }^{3}$, Martin Esmann', Simon F. Becker ${ }^{2}$, Ralf Vogelgesang ${ }^{2}$, Peter A. van Aken ${ }^{1}$

1. Max Planck Institute for Solid State Research, Stuttgart, Germany

2. Carl von Ossietzky University of Oldenburg, Oldenburg, Germany

3. Laboratoire de Physique des Solides, Université Paris Sud, Orsay 91400, France

Plasmonic metallic tapers with conical shapes are one of the most common and simple structures, with concomitant capabilities of nanofocusing and strong field enhancement. Such tapers can serve either as waveguides or nanoantennas in near-field scanning optical microscopy and as a ultrafast photoemission point source in electron microscopy [1]. Electron energy-loss spectroscopy (EELS) is exploited to comprehensively study the plasmonic response of mesoscopic tapers in a wide energy range. However, the interpretation of EELS signatures is intricate by the fact that different resonance mechanisms are active when relativistic electrons interact with mesoscopic plasmonic tapers, namely phase matching [2] and reflection [3] (Fig.1a). It is of fundamental importance to clarify the reasons behind the EELS resonances explicitly.

We systematically investigated the plasmonic modes of gold tapers with various opening angles from $5^{\circ}$ to $47^{\circ}$ both experimentally and theoretically by using both EELS and numerical finite-difference timedomain (FDTD) simulations. Single crystalline gold tapers with very smooth surfaces were fabricated at Oldenburg University by electrochemical etching in $\mathrm{HCl}$ [4]. The tapers were mounted on a 3-mm copper ring for TEM analysis. EELS experiments were conducted at the Zeiss SESAM microscope operated at an acceleration voltage of $200 \mathrm{kV}$. EELS data were acquired with an energy resolution of 90 $\mathrm{meV}$ as determined from the full width at half-maximum of the zero-loss peak (ZLP). The acquisition time of each spectrum was in the range between 0.2 and $0.8 \mathrm{~s}$.

As shown in Fig.1 e-g, distinct resonances along the taper shaft are observed in tapers independent of opening angles. We show that, despite their similarity, the formation of these resonances is from a competition between two coexisting mechanisms (Fig.1 b-d). Phase matching between the field components of the fast electron and of higher-order angular momentum modes of the taper is the dominant contribution to the electron energy loss when the interaction length between the swift electron and the taper near-field becomes large enough, so that the higher-order angular momentum orders are excited. In contrast, reflection from the taper apex dominates the EELS contrast in gold tapers when mostly the fundamental $m=0$ mode is excited. A gradual transition of these two mechanisms is observed.

In addition to EELS, which measures extinction, cathodoluminescence [5] has been applied to study the mere radiative behavior of the excited plasmons of gold tapers. One can see that the excited plasmons at both the apex and the shaft (Fig.2b) are coupled to the far-field radiation (Fig. 2a).

In conclusion, we disentangle the link and distinction between the two different dynamic mechanisms, reflection and phase matching, of surface plasmons excited by relativistic electrons in a threedimensional single-crystalline gold taper [6]. 
References:

[1] S.Guo et al., Nano Lett. 16 (2016), p.6137.

[2] N. Talebi et al., ACS Nano 9 (2015), p.7641.

[3] B. Schroeder et al., Phys. Rev. B 92 (2015), p.085411.

[4] S. Schmidt et al., ACS Nano 6 (2012) p.6040.

[5] M. Kociak and L.F. Zagonel, Ultramicroscopy 174 (2017), p.50.

[6] The research leading to these results has received funding from the European Union Seventh Framework Programme [FP7/2007-2013] under grant agreement No. 312483 (ESTEEM2).
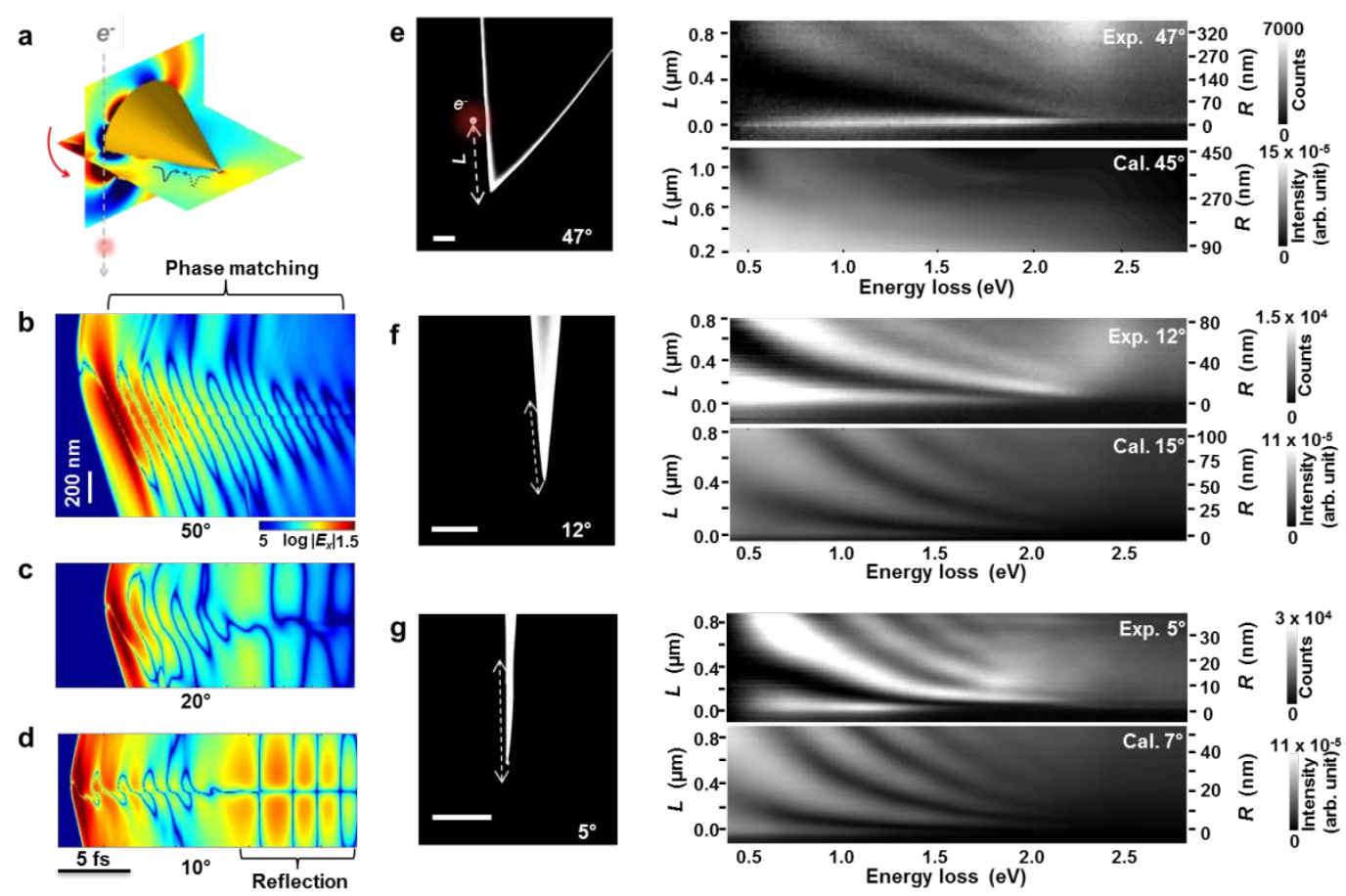

Figure 1. (a) Coexisting interaction mechanisms of phase matching and reflection when a relativistic electron is passing by a taper surface, indicated by red and blue arrows, respectively. (b-d) Transition from phase matching to reflection revealed by numerically calculating total scattered electric field along the electron trajectory versus electron trajectory (nm) and time (fs) for tapers with different opening angles of $50^{\circ}, 20^{\circ}$ and $10^{\circ}$ at a constant distance of $L=1460 \mathrm{~nm}$ from the apex. (e-g) Dark-field imaging and spectroscopy of gold tapers with full opening angles of $47^{\circ}, 12^{\circ}$, and $5^{\circ}$, respectively. Both experimental and computed EELS are plotted as a function of impact location along the taper shaft and the corresponding local radius. Scale bar lengths for $(\mathrm{e}-\mathrm{g})$ are $500 \mathrm{~nm}$. Adapted with kind permission from [1]. Copyright (2016) American Chemical Society.
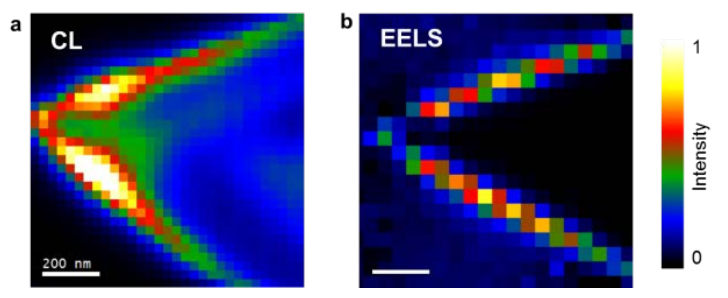

Figure 2. (a) CL and (b) EELS images of a taper with an opening angle of $47^{\circ}$ at the same energy loss of $1.91 \mathrm{eV}$. Scale bar lengths are $200 \mathrm{~nm}$. 